\title{
The effect of Dehydroepiandrosterone on insulin resistance in patients with impaired glucose tolerance
}

\author{
Afsaneh Talaei ${ }^{1}$, Masoud Amini², Mansour Siavash ${ }^{2}$, Maryam Zare ${ }^{3}$ \\ ${ }^{1}$ Arak University of Medical Sciences, ${ }^{2}$ Isfahan University of Medical Sciences, ${ }^{3}$ Isfahan Endocrine \& Metabolism \\ Research Center, Isfahan, Iran
}

\begin{abstract}
OBJECTIVE: Dehydroepiandrosterone (DHEA) and Dehydroepiandrosterone-sulfate (DHEAS) are the most abundant steroid hormones in the body. Recently, DHEA-S has gained interest as an antidepressant substance, with positive effects on autoimmune disease such as lupus and ulcerative colitis, as well as obesity, cancer, cardiovascular disease and diabetes. Its effect on insulin resistance is also assumed to be positive, but has not as yet been confirmed. The present cross-over clinical trial was conducted to evaluate the efficacy of DHEA and placebo on insulin resistance. DESIGN: Participants were selected among relatives of diabetic patients who were referred to the Isfahan Endocrine Research Center because of Impaired Glucose Tolerance (IGT) test. Thirty IGT patients were treated randomly with DHEA $(50 \mathrm{mg} / \mathrm{day})$ or placebo by cross-over clinical trial for six months and insulin resistance between the beginning and the end of each three months treatment period was assessed. RESULTS: At the end of the first three months, the mean changes from baseline of the various parameters in the drug group were: DHEA-S, $2.5 \mu \mathrm{mol} / \mathbf{l}(\mathrm{p}=\mathbf{0 . 0 0 8})$; Homeostatic Model Assessment of Insulin Resistance (HOMA-IR), $0.6(p=0.6)$; insulin, 7.1 pmol/1 $(p=0.3)$ and FPG, $0.5 \mathrm{mmol} / \mathrm{l}(\mathrm{p}=0.1)$. The changes in the placebo group were: DHEA-S, $0.08 \mu \mathrm{mol} / \mathrm{l}(\mathrm{p}=0.6)$; HOMA-IR, $0.9(p=0.03)$; FPG, 0.8 $\mathrm{mmol} / \mathrm{l}(\mathrm{p}=0.1)$; insulin, $25.1 \mathrm{pmol} / \mathrm{l}(\mathrm{p}=0.05)$. In the second three months, the mean changes in the drug group were: DHEA-S, $4.5 \mu \mathrm{mol} / \mathrm{l}(\mathrm{p}=0.003)$; Fasting Plasma Glucose (FPG), 0.1 $\mathrm{mmol} / \mathrm{l}(\mathrm{p}=0.4)$; insulin, $4.3 \mathrm{pmol} / \mathrm{l}(\mathrm{p}=0.2)$; HOMA-IR, $0.3(\mathrm{p}=0.1)$ and the changes in placebo group were: DHEA-S, $0.7 \mu \mathrm{mol} / \mathrm{l}(\mathrm{p}=0.5)$; FPG, $0.3 \mathrm{mmol} / \mathrm{l}(\mathrm{p}=0.3)$; insulin, $10.7 \mathrm{pmol} / \mathrm{l}$ $(p=0.1)$; HOMA-IR, $0.6(p=0.03)$. CONCLUSION: DHEA did not reduce insulin resistance, although there was a tendency to improvement. The data indicate a possible but not clearly favorable effect of DHEA on insulin resistance.
\end{abstract}

Keywords: DHEA, Dehydroepiandrosterone-sulfate, Diabetes mellitus, Glucose intolerance, Insulin resistance 


\section{INTRODUCTION}

Steroids were first described by Butenandt in 1934 . Dehydroepiandrosterone (DHEA) and its sulfated metabolite Dehydroepiandrosterone Sulfate (DHEAS) are the most abundant circulating adrenal steroids in humans. DHEA-S, in contrast to other adrenal hormones, decreases with advancing age, so that it is at $20 \%$ of its peak in the seventies. It is therefore called the fountain of youth. ${ }^{1}$ The decrease in DHEA$\mathrm{S}$ is suspected to be related to mental and physical disabilities in elder people. ${ }^{2}$ In the USA, DHEA is considered a food supplement rather than a drug. It is a hormone not necessary for life but affects different tissues through conversion to estrogens and more active androgens. ${ }^{3}$

DHEA-S has gained interest as an antidepressant substance, with positive effects on autoimmune disease such as lupus and ulcerative colitis, as well as obesity, cancer, cardiovascular disease and diabetes. ${ }^{4}$ Its effect on insulin resistance is also assumed to be positive, but has not as yet been confirmed. Certain studies indicated significant effects of DHEA-S on body composition and glucose tolerance. Thus, Lasco et al demonstrated that the intake of DHEA for one year improved insulin sensitivity as well as Triglyceride (TG) and cholesterol levels. ${ }^{5}$ Dhatariya et al found that administration of DHEA for 12 weeks to women with adrenal insufficiency decreased insulin and SHBG levels, while it did not affect body composition. DHEA was also found to decrease total cholesterol, TG and LDL cholesterol. Glucose levels were also found lower in the group treated with DHEA. ${ }^{2}$

According to another study, ${ }^{6}$ benfluorex caused a reduction in insulin and a rise in DHEA values. Specifically, DHEA rose from 6.8 to $10.5 \mu \mathrm{mol} / \mathrm{ml}$ and DHEA-S from 13.6 to $22.7 \mu \mathrm{mol} / \mathrm{ml}$. In another study, DHEA improved pancreatic function and increased beta-cell mass and insulin secretion in animals. ${ }^{7}$ Formoso et al found that these effects were associated with increased levels of nitric oxide and endothelin $-1{ }^{8}$

DHEA-S does not necessarily increase insulin directly. It might increase mRNA in mitochondria and secretive response of insulin to glucose. ${ }^{9}$ One potential mechanism by which DHEA improves insulin resistance is a decrease in TNF-alpha level and in body weight. ${ }^{10}$ On the other hand, some studies have presented different results pointing out the negative role of DHEA-S in diabetes. According to a study by Saruc et al, DHEA can cause the onset of diabetes and obesity in women at menopause. ${ }^{11}$ Higher levels of DHEA-S were associated with insulin resistance in women younger than 35 years old. ${ }^{12}$ Liu et al demonstrated that DHEA-S deteriorated glucose control and beta-cell function in vitro. ${ }^{13}$ Due to the divergent results concerning the effects of DHEA-S on diabetes and especially insulin resistance, we performed a study to assess the effect of DHEA on insulin resistance and consequently on the glycemic control in patients with impaired glucose tolerance.

\section{PATIENTS AND METHODS}

The study was designed as a cross-over randomized clinical trial. The participants were female relatives of diabetic patients. Based on the results of an Oral Glucose Tolerance Test (OGTT), 30 females, relatives of women with type 2 diabetes referred to the Isfahan Endocrine Research Center because of Impaired Glucose Tolerance (IGT) were included in the study. The patients were randomly assigned into two groups with 15 patients per group. The mean age, weight and Body Mass Index (BMI) in the drug group were 40.6 years, $70.8 \mathrm{Kg}$ and $30 \mathrm{~kg} / \mathrm{m}^{2}$, respectively. The mean age, weight and BMI in the placebo group were 41 years, $75.1 \mathrm{Kg}, 32.3 \mathrm{~kg} / \mathrm{m}^{2}$, respectively. These baseline parameters were comparable in the two groups $(\mathrm{p}=0.1)$. Women in menopause in the drug and placebo groups were $8 \%$ and $10 \%$, respectively. The methodology, and the potential side-effects, were explained to the participants. Written consent was obtained from those who agreed to participate. The study was approved by the Ethics Committee of the Isfahan Endocrine and Metabolism Research Center of Isfahan University of Medical Sciences.

In the next step, a trained physician interviewed the patients, examined them and filled in a questionnaire. The questionnaire included information on age, gender, occupation, education level, family history of diabetes, drug history, medical history and menopausal status. Physical examination included records of height, weight, BMI, waist circumference and blood pressure. Fasting plasma samples were then obtained for blood glucose, HbA1c, cholesterol, Low 
Density Lipoprotein cholesterol (LDL), High Density Lipoprotein cholesterol (HDL), triglycerides, Blood Urea Nitrogen (BUN), creatinine, insulin, DHEA-S, Liver function tests, Bil-T, Bil-D, and Complete Blood Count with differential. In addition, homeostatic model assessment for insulin resistance (HOMA-IR) was calculated using the following formula:

$$
\begin{aligned}
& \text { HOMA-IR }=\text { Insulin }(\mu \mathrm{U} / \mathrm{ml}) \\
& \times \text { glucose }(\mathrm{mmol} / \mathrm{L}) / 22.5
\end{aligned}
$$

During the first trimester of the study, either DHEA $50 \mathrm{mg}$ or placebo was given daily to the drug group and the control group, respectively. At the end of the first three months, the patients were re-evaluated by the use of the above-mentioned questionnaire. Physical examination and serum biochemistry (insulin, fasting plasma glucose, liver function tests and lipid profile) were also determined and Homeostatic Model Assessment of Insulin Resistance (HOMAIR) was estimated.

After a two-week wash-out period, the second trimester study period was initiated and the same parameters were assessed. According to the cross-over design, the placebo group received DHEA and the DHEA group received placebo during this period. Finally, the results of both groups were compared and the relation of HOMA-IR to the DHEA-S levels was evaluated with the paired sample t-test. The results were analyzed using SPSS 12.

\section{RESULTS}

At the end of the first trimester in the drug group the changes from baseline were as follows: mean $\pm \mathrm{SD}$ DHEA-S values increased from $3.1 \pm 1.7$ to $5.7 \pm 3.2$ $\mu \mathrm{mol} / 1$ (116 \pm 66 to $211 \pm 119 \mu \mathrm{g} / \mathrm{dl})$ and HOMA-IR from $1.8 \pm 0.9$ to $2.4 \pm 1$.4. Insulin values increased from $62.4 \pm 26.5$ to $70.3 \pm 24.3 \mathrm{pmol} / 1(8.7 \pm 3.7$ to $9.8 \pm 3.4$ $\mu \mathrm{IU} / \mathrm{ml})$ and blood glucose from $5 \pm 0.4$ to $5.3 \pm 1.6$ $\mathrm{mmol} / \mathrm{l}(87 \pm 7$ to $97 \pm 29 \mathrm{mg} / \mathrm{dl})$. In the placebo group, mean \pm SD DHEA-S values changed from $2.6 \pm 1$. 1 to $2.5 \pm 1.3 \mu \mathrm{mol} / 1(97 \pm 44$ to $94 \pm 48 \mu \mathrm{g} / \mathrm{dl})$ and HOMAIR from $1.2 \pm 0.7$ to $2.1 \pm 0.9$. Insulin levels increased from $45.9 \pm 23.6$ to $71 \pm 64.5 \mathrm{pmol} / 1(6.4 \pm 3.3$ to $9.9 \pm 9$ $\mu \mathrm{IU} / \mathrm{ml})$ and the blood glucose from $4.6 \pm 0.5$ to $5.5 \pm 1.6$ $\mathrm{mmol} / \mathrm{l}(84 \pm 9$ to $100 \pm 29 \mathrm{mg} / \mathrm{dl})$ (Table 1$)$. The mean difference from baseline in the drug group was for DHEA-S $2.5 \mu \mathrm{mol} / \mathrm{l}(95 \mu \mathrm{g} / \mathrm{dl})(\mathrm{p}=0.008)$, HOMAIR $0.62(p=0.6)$, Fasting Plasma Glucose (FPG) 0.5 $\mathrm{mmol} / \mathrm{l}(10 \mathrm{mg} / \mathrm{dl})(\mathrm{p}=0.1)$ and insulin $7.1 \mathrm{pmol} / \mathrm{l}(1$ $\mu \mathrm{IU} / \mathrm{ml})(\mathrm{p}=0.3)$. The mean difference in the placebo group was for DHEA-S $0.08 \mu \mathrm{mol} / \mathrm{l}(3 \mu \mathrm{cg} / \mathrm{dl})$ $(\mathrm{p}=0.6)$, HOMA-IR $0.9(\mathrm{P}=0.03)$, FPG $0.8 \mathrm{mmol} / 1$ $(16 \mathrm{mg} / \mathrm{dl})(\mathrm{p}=0.1)$ and insulin level $25.1 \mathrm{pmol} / \mathrm{l}(3.5$ $\mu \mathrm{IU} / \mathrm{ml})(\mathrm{p}=0.05)$.

Comparison between the beginning of the first trimester in the drug group and the beginning of the second trimester in the placebo group (washout) demonstrated that there was no difference in any of the variables except the values of FPG which differed significantly $5 \pm 0.4$ vs $6 \pm 0.9 \mathrm{mmol} / 1(87 \pm 7$ vs. $108 \pm 17 \mathrm{mg} / \mathrm{dl})(\mathrm{p}=0.02)$.Comparison between the beginning of the first trimester in the placebo group and the beginning of the second trimester in the drug group showed that only the insulin differed significantly 45.9 vs 58.8 pmol/1 (6.4 vs. $8.2 \mu \mathrm{IU} / \mathrm{ml})$, $\mathrm{p}=0.001$ (Table 2).

At the end of the second trimester in the drug group, mean \pm SD values of DHEA-S increased from

Table 1. Comparison* of variables between the beginning and end of the first trimester in the drug and placebo groups. Values are expressed

\begin{tabular}{|c|c|c|c|c|c|c|}
\hline \multirow[b]{2}{*}{ Variable } & \multicolumn{3}{|c|}{ Placebo group } & \multicolumn{3}{|c|}{ Drug group } \\
\hline & Basal & End of first trimester & P-value & Basal & End of first trimester & P-value \\
\hline$\overline{\mathrm{FPG}(\mathrm{mmol} / \mathrm{l})}$ & $4.6 \pm 0.5$ & $5.5 \pm 1.6$ & 0.1 & $5 \pm 0.4$ & $5.3 \pm 1.6$ & 0.1 \\
\hline Insulin (pmol/l) & $45.9 \pm 23.6$ & $71 \pm 64.5$ & 0.05 & $62.4 \pm 26.5$ & $70.3 \pm 24.3$ & 0.3 \\
\hline HOMA-IR & $1.2 \pm 0.7$ & $2.1 \pm 0.9$ & 0.03 & $1.8 \pm 0.9$ & $2.4 \pm 1.4$ & 0.6 \\
\hline DHEA-S $(\mu \mathrm{mol} / \mathrm{l})$ & $2.6 \pm 1.1$ & $2.5 \pm 1.3$ & 0.6 & $3.1 \pm 1.7$ & $5.7 \pm 3.2$ & 0.008 \\
\hline
\end{tabular}
as means $\pm \mathrm{SD}$

*The paired samples t-test was applied.

FPG: Fasting Plasma Glucose, HOMA-IR: Homeostatic Model Assessment - Insulin Resistance, DHEAS: Dehydroepiandrosterone Sulfate 
Table 2. Comparison* of variables following the wash-out period between the beginning of the first trimester and the beginning of the second trimester in the DHEAS and placebo groups. Values are expressed as means \pm SD.

\begin{tabular}{|c|c|c|c|c|c|c|}
\hline & Placebo & Drug & & Drug & Placebo & \\
\hline Variable & $\begin{array}{l}\text { Beginning of the } \\
\text { first trimester }\end{array}$ & $\begin{array}{l}\text { Beginning of the } \\
\text { second trimester }\end{array}$ & P-value & $\begin{array}{l}\text { Beginning of the } \\
\text { first trimester }\end{array}$ & $\begin{array}{l}\text { Beginning of the } \\
\text { second trimester }\end{array}$ & P-value \\
\hline $\mathrm{FPG} \mathrm{(mmol/l)}$ & $4.6 \pm 0.5$ & $5 \pm 1$ & 0.8 & $5 \pm 0.4$ & $6 \pm 0.9$ & 0.02 \\
\hline Insulin (pmol/l) & $45.9 \pm 23.6$ & $58.8 \pm 15$ & 0.001 & $62.4 \pm 26.5$ & $86.8 \pm 25.1$ & 0.1 \\
\hline HOMA-IR & $1.2 \pm 0.7$ & $1.7 \pm 0.5$ & 0.4 & $1.8 \pm 0.9$ & $3 \pm 1.3$ & 0.3 \\
\hline DHEA-S $(\mu \mathrm{mol} / \mathrm{l})$ & $2.6 \pm 1.1$ & $3.1 \pm 1.6$ & 0.3 & $3.1 \pm 1.7$ & $4.2 \pm 2.2$ & 0.5 \\
\hline
\end{tabular}

* The paired samples t-test was applied.

FPG: Fasting Plasma Glucose, HOMA-IR: Homeostatic Model Assessment - Insulin Resistance, DHEAS: Dehydroepiandrosterone Sulfate

$3.1 \pm 1.6$ to $7.6 \pm 2.3 \mu \mathrm{mol} / \mathrm{l}(117 \pm 62$ to $283 \pm 88 \mu \mathrm{cg} / \mathrm{dl})$, of FPG from $5 \pm 1$ to $5.1 \pm 0.6 \mathrm{mmol} / \mathrm{l}(89 \pm 18$ to $92 \pm 11.2$ $\mathrm{mg} / \mathrm{dl}$ ), of insulin from $58.8 \pm 15$ to $63.1 \pm 17.2 \mathrm{pmol} / \mathrm{l}$ $(8.2 \pm 2.1$ to $8.8 \pm 2.4 \mu \mathrm{IU} / \mathrm{ml})$ and of HOMA-IR from $1.7 \pm 0.5$ to $2 \pm 0.6$. In the placebo group, mean \pm SD values of DHEA-S changed from $4.2 \pm 2.2$ to $3.5 \pm 2.6$ $\mu \mathrm{mol} / \mathrm{l}(156 \pm 82.7$ to $130 \pm 97 \mu \mathrm{cg} / \mathrm{dl})$, of FPG from $6 \pm 0.9$ to $5.6 \pm 0.6 \mathrm{mmol} / \mathrm{l}(108 \pm 17$ to $102 \pm 12 \mathrm{mg} / \mathrm{dl})$, of insulin from $86.1 \pm 25.1$ to $75.3 \pm 2.8 \mathrm{pmol} / 1(12 \pm 3.5$ to $10.5 \pm 0.4 \mu \mathrm{IU} / \mathrm{ml}$ ) and of HOMA-IR from $3 \pm 1.3$ to $2.4 \pm 1.1$. For $p$ values see Table 3 .

\section{DISCUSSION}

The results of the present study demonstrate that DHEA possibly has a beneficial effect on insulin resistance. Thus, our results at the end of the first trimester demonstrated that HOMA-IR in the drug group did not significantly increase (from 1.8 to 2.4, $\mathrm{p}=0.6$ ), while in the placebo group they increased significantly $(p=0.03)$. The insulin levels also significantly increased in the placebo group, while no significant alterations were observed in the drug group. As expected, DHEA-S values increased in the drug group but not in the placebo group. After the two-week wash-out period, considered sufficient to eliminate the previous effect of DHEA in the drug group, the women changed group.

At the end of the second trimester of the study, DHEA-S increased from 3.1 to $7.6 \mu \mathrm{mol} / 1$ (117 to $283 \mu \mathrm{cg} / \mathrm{dl})$ in the drug group and did not significantly change in the placebo group. The changes in insulin values in both groups were not significant, but HOMA-IR decreased significantly in the placebo group, which is not easy to explain.

In other words, patients who took DHEA in the first trimester decreased insulin resistance in the second trimester when they were on placebo. This observation might indicate that the wash-out period was not sufficient or that the effects of DHEA lasted longer even after discontinuation of the drug. The first trimester results are more clear-cut, indicating that daily DHEA supplementation to women with IGT during a three-month period favorably affected insulin sensitivity.

Table 3. Comparison of variables between the beginning and end of the second trimester in the drug and placebo groups (by paired samples $\mathrm{t}$-test), values are expressed as means $\pm \mathrm{SD}$

\begin{tabular}{|c|c|c|c|c|c|c|}
\hline \multirow[b]{2}{*}{ Variable } & \multicolumn{3}{|c|}{ Placebo } & \multicolumn{3}{|c|}{ Drug } \\
\hline & Beginning & End & P-value & Beginning & End & P-value \\
\hline $\mathrm{FPG}(\mathrm{mmol} / \mathrm{l})$ & $6 \pm 0.9$ & $5.6 \pm 0.6$ & 0.3 & $5 \pm 1$ & $5.1 \pm 0.6$ & 0.4 \\
\hline Insulin $(\mathrm{pmol} / \mathrm{l})$ & $86.1 \pm 25.1$ & $75.3 \pm 2.8$ & 0.1 & $58.8 \pm 15$ & $63.1 \pm 17.2$ & 0.2 \\
\hline HOMA-IR & $3 \pm 1.3$ & $2.4 \pm 1.1$ & 0.03 & $1.7 \pm 0.5$ & $2 \pm 0.6$ & 0.1 \\
\hline DHEA-S $(\mu \mathrm{mol} / \mathrm{l})$ & $4.2 \pm 2.2$ & $3.5 \pm 2.6$ & 0.5 & $3.1 \pm 1.6$ & $7.6 \pm 2.3$ & 0.003 \\
\hline
\end{tabular}

FPG: Fasting Plasma Glucose, HOMA-IR: Homeostatic Model Assessment - Insulin Resistance, DHEAS: Dehydroepiandrosterone Sulfate 
One could mention certain mechanisms which possibly explain the effects of DHEA-S. Metabolism and secretion of many steroid hormones are altered in diabetes. Thus, in cases of poor glycemic control in both type 1 diabetes ${ }^{14}$ and also in type 2 diabetes, ${ }^{15}$ DHEA and DHEA-S are decreased. Furthermore DHEA has been shown to increase the number of pancreatic beta-cells and improve glycemic control by increasing insulin release in animals.

In a study by Kawano et al DHEA improved endothelial function and insulin resistance,${ }^{16}$ while in another study in type 2 diabetes, a strong inverse relation between insulin levels and atherosclerosis was observed..$^{15}$ According to a study in Japan, the development of type 2 diabetes in men is associated with decreasing DHEA-S levels. ${ }^{17}$ In another study, it was shown that taking DHEA for one year could improve insulin resistance and potentially prevent the development of diabetes. ${ }^{5}$

An inverse relation between DHEA-S levels and severity of albuminuria has also been reported. DHEA$\mathrm{S}$ may be the link for the association of increasing albuminuria with higher cardiovascular mortality. ${ }^{18}$ In another study, a two-week intake of DHEA by type 2 diabetic men improved glucose control and HOMA-IR.${ }^{19}$ Other results however have shown that a two year intake of DHEA had no effect on insulin sensitivity ${ }^{3,20}$ and quality of life. ${ }^{20}$

In a study by Yamauchi et al, it was found that in diabetic men DHEA levels were significantly lower compared to men with IGT but DHEA levels were not related to insulin levels. ${ }^{21}$ Analogous results were found by Jedrzejuk et al who studied the effect of DHEA intake for three months and found that this treatment had no effect on cholesterol, triglyceride and insulin sensitivity. ${ }^{22}$

Our data indicate a possible but no clearly favorable effect of DHEA on insulin resistance.

In general, there are contradictory results with regard to DHEA and DHEA-S effects on insulin resistance. The differences may be caused by variations in sample size, treatment duration, treatment dose and population sample. Therefore, further studies with large sample sizes of suitable duration are needed to clarify whether or not DHEA and DHEA-S have a favorable effect on diabetic control.

\section{REFERENCES}

1. Baulieu EE, 1996 Dehydroepiandrosterone (DHEAS): A Fountain of youth? J Clin Endocrinol Metab 81: 3147-3151.

2. Dhatariya K, Bigelow ML, Nair KS, 2005 Effect of Dehydroepiandrosterone Replacement on Insulin Sensitivity and Lipids in Hypoadrenal Women. Diabetes 54: 765-769.

3. Nair KS, Rizza RA, Brien P, et al, 2006 DHEA-S in elderly women and DHEA-S or testosterone in elderly men. N Engl J Med 355: 1647-1659.

4. Arlt W, 2006 Dehydroepiandrosterone replacement therapy. Current Opinion in Endocrinology \& Diabetes 13: 291-305.

5. Lasco A, Frisina N, Morabito N, et al, 2001 Metabolic effects of DHEA-S therapy in post menopausal women. Eur J Endocirol 145: 457-461.

6. Nestler JE, Beer NA, Jacobowicz DJ, Colombo C, Beer RM, 1996 Effects of Insulin Reduction with Benfluorex on Serum DHEA-S and Blood Pressure in Hypertensive Middle-Aged and Elderly Men. J Clin Endocrinol Metab 80: 700-706.

7. Medina MC, Souza LC, Caperuto LC, et al, 2006 Dehydroepiandrosterone increases beta-cell mass and improves the glucose-induced insulin secretion by pancreatic islets from aged rats. FEBS Lett 580: 285290.

8. Formoso G, Chen H, Kim J, Montagnani M, Consoli A, Quon M, 2006 Dehydroepiandrosterone Mimics Acute Actions of Insulin to Stimulate Production of Both Nitric Oxide and Endothelin 1 via Distinct Phosphatidylinositol3-Kinase- and Mitogen-Activated Protein Kinase- Dependent Pathways in Vascular Endothelium. J Molecular Endocrinology 20: 1153-1163.

9. Dillon J, Yaney G, Zhou Y, et al, 2000 Dehydroepiandrosterone Sulfate and B-Cell Function Enhanced Glucose-Induced Insulin Secretion and Altered Gene Expression in Rodent Pancreatic B-Cells. J Diabetes 49: 2012-2020.

10. Kimura M, Tanaka SI, Yamada Y, Kiuchi Y, Yamakawa T, Sekihara H, 1998 Dehydroepiandrosterone decreases serum tumor necrosis factor and restores insulin sensitivity. J Endocrinology 139: 3249-3253.

11. Saruç M, Yüceyar H, Ayhan S, Türkel N, Tuzcuoglu I, Can M, 2003 The association of dehydroepiandrosterone, obesity, waist-hip ratio and insulin resistance with fatty liver in postmenopausal women, a hyperinsulinemic euglycemic insulin clamp study. Hepatogastroenterology 50: 771 .

12. Mino D, Amato D, Cuevas ML, et al, 2002 A relationship of insulin resistance and overweight with cortisol and dehydroepiandrosterone-sulfate levels. Arch Med 
Res 33: 524-530.

13. Liu HK, Green BD, McClenaghan NH, McCluskey JT, Flatt PR, 2006 Deleterious effects of supplementation with dehydroepiandrosterone sulphate or dexamethasone on rat insulin-secreting cells under in vitro culture condition. Biosci Rep 26: 31-38.

14. Couch RM, 1992 Dissociation of cortisol and adrenal androgen secretion in poorly controlled insulin-dependent diabetes mellitus. Acta Endocrinologica 127: 115-117.

15. Fukui M, Kitagawa Y, Nakamura N, et al, 2005 Serum dehydroepiandrosterone sulfate concentration and carotid atherosclerosis in men with type 2 diabetes. Atherosclerosis 181: 339-344.

16. Kawano H, Yasue H, Kitagawa A, et al, 2003 Dehydroepiandrosterone Supplementation Improves Endothelial Function and Insulin Sensitivity in Men. J Clin Endocrinol Metab 88: 3190-3195.

17. Kameda W, Daimon M, Oizumi T, et al, 2005 Association of decrease in serum dehydroepiandrosterone sulfate levels with the progression to type 2 diabetes in men of a Japanese population: the Funagata Study. J Metab 54: 669-676.

18. Fukui M, Kitagawa Y, Nakamura N, Kadono M, Hasegawa G, Yoshikawa T, 2004 Association between urinary albumin excretion and serum dehydroepiandrosterone sulfate concentration in male patients with type 2 diabetes: a possible link between urinary albumin excretion and cardiovascular disease. Diabetes Care 2712: 2893-2897.

19. Yamada Y, Sekihara H, Omura M, et al, 2007 Changes in serum sex hormone profiles after short term low dose administration of DHEA-S to young and elderly persons. J Endocrine 54: 153-162.

20. Basu R, Dalla Man C, Campioni M, et al, 2007 Two years of treatment with dehydroepiandrosterone does not improve insulin secretion, insulin action, or postprandial glucose turnover in elderly men or women. Diabetes: 753-766.

21. Yamauchi A, Takei I, Kasuga A, et al, 1996 Depression of dehydroepiandrosterone in Japanese diabetic men comparison between non-insulin-dependent diabetes mellitus and impaired glucose tolerance. Eur J Endocrinol 135: 101-104.

22. Jedrzejuk D, Medras M, Milewicz A, Demissie M, 2003 Dehydroepiandrosterone replacement in healthy men with age-related decline of DHEA-S-S: effects on fat distribution, insulin sensitivity and lipid metabolism. J Aging Male 6: 151-156. 